\title{
Silver Nanoparticles and Mitochondrial Interaction
}

\author{
Eriberto Bressan, ${ }^{1}$ Letizia Ferroni, ${ }^{2}$ Chiara Gardin, ${ }^{2}$ Chiara Rigo, ${ }^{3}$ Michele Stocchero, \\ Vincenzo Vindigni, ${ }^{1}$ Warren Cairns, ${ }^{3}$ and Barbara Zavan ${ }^{2}$ \\ ${ }^{1}$ Department of Biomedical Sciences, University of Padova, Via G. Colombo 3, 35100 Padova, Italy \\ ${ }^{2}$ Department of Neurosciences, University of Padova, Via Venezia 90, 35100 Padova, Italy \\ ${ }^{3}$ CNR-IDPA c/o Department of Environmental Sciences Informatics and Statistics, Universita Ca' Foscari, Dorsoduro 2137, \\ 30123 Venezia, Italy
}

Correspondence should be addressed to Barbara Zavan; barbara.zavan@unipd.it

Received 14 May 2013; Revised 10 July 2013; Accepted 31 July 2013

Academic Editor: Ryo Jimbo

Copyright (C) 2013 Eriberto Bressan et al. This is an open access article distributed under the Creative Commons Attribution License, which permits unrestricted use, distribution, and reproduction in any medium, provided the original work is properly cited.

Nanotechnology has gone through a period of rapid growth, thus leading to the constant increase in the application of engineered nanomaterials in daily life. Several different types of nanoparticles have been engineered to be employed in a wide array of applications due to their high surface to volume ratio that leads to unique physical and chemical properties. So far, silver nanoparticles (AgNps) have been used in many more different medical devices than any other nanomaterial, mainly due to their antimicrobial properties. Despite the promising advantages posed by using AgNps in medical applications, the possible health effects associated with the inevitable human exposure to AgNps have raised concerns as to their use since a clear understanding of their specific interaction with biological systems has not been attained yet. In light of such consideration, aim of the present work is the morphological analysis of the intracellular behavior of AgNps with a diameter of $10 \mathrm{~nm}$, with a special attention to their interaction with mitochondria.

\section{Introduction}

Antibacterial properties of silver ions are well known. Indeed, silver has been used since time immemorial in different chemical forms to treat burns, wounds, and several different infections caused by pathogenic bacteria. Interestingly, for thousands of years, silver and silver ions have been used for their bactericidal properties $[1,2]$, which include

(1) multilevel antibacterial effects that considerably reduce the chances of developing resistance since this effect of silver is thought to be due to blockage of respiratory enzyme pathways and alteration of microbial DNA and the cell wall $[3,4]$;

(2) effectiveness against multidrug-resistant organisms $[5,6]$;

(3) low systemic toxicity [7, 8].
Over the past decade, a variety of advanced silver-based medical devices have been developed with considerable variations in the structure, composition, and silver content. In recent years, nanotechnology has provided the means of producing pure silver nanoparticles, markedly increasing the rate of silver ion release and its antimicrobial activity as well.

The use of oral implants in the rehabilitation of partially and fully edentulous patients is widely accepted even though failures do occur [9]. The chance for implants to integrate can for example be jeopardised by the intraoral presence of bacteria and concomitant inflammatory reactions. The longevity of osseointegrated implants can be compromised by occlusal overload and/or plaque-induced peri-implantitis, depending on the implant geometry and surface characteristics. Animal studies, cross-sectional and longitudinal observations in man, and association studies indicate that peri-implantitis is characterised by a microbiota comparable to that of periodontitis (high proportion of anaerobic 
Gram-negative rods, motile organisms, and spirochetes), but this does not necessarily prove a causal relationship [10]. However, in order to prevent such a bacterial shift, the following measures can be considered: periodontal health in the remaining dentition (to prevent bacterial translocation), the avoidance of deepened peri-implant pockets, and the use of a relatively smooth abutment and implant surface. Finally, periodontitis enhancing factors such as smoking and poor oral hygiene also increase the risk for peri-implantitis [11].

The oral cavity is populated by a variety of microorganisms. The microbial communities in the oral cavity are polymicrobial and exist primarily as biofilms [12]. These biofilms can be responsible for several local diseases, including periodontal and peri-implant diseases, which can lead to the loss of teeth or implants, respectively. The potential of silver nanoparticles (AgNps) to reduce bacterial adhesion to dental implant surfaces and to prevent biofilm formation has been investigated by many authors $[13,14]$ with a view to reducing the risk of peri-implant infections. Another interesting application of Ag NPs in dentistry pertains the structural and surface modification of bone grafts and membranes with a view to preventing the risk of contamination and associated infection that are common when bone augmentation techniques such as guided bone regeneration (GBR) and guided tissue regeneration (GTR) are used [15-19].

Despite the widespread use of Ag NPs, a lack of information on their biological effects on human cells and environments still exists. Some authors have investigated the potential toxicity of Ag NPs in different cell systems, including bacteria and mammalian cells. Such studies have attributed the cytotoxicity of Ag NPs to several possible mechanisms, including the dissolving or release of Ag ions from the nanoparticles, the disruption of cell membrane integrity, oxidative stress, protein or DNA binding and damage, the generation of reactive oxygen species, and apoptotic cell death. The toxic mechanism seems likely to depend on the nanoparticles' properties too, for example surface area, size and shape, capping agent, surface charge, particle purity, structural distortion, and the bioavailability of the individual particles. To this view, in previous works, our group compared the silver structure and content of several Ag dressing based products, focusing main attention on Acticoat $[20,21]$.

Acticoat is a nanocrystalline silver dressing composed of two layers of silver-coated high-density polyethylene, enclosing a rayon/polyester core of apertured nonwoven fabric. The elements that compose Acticoat are welded together ultrasonically. When moistened with water, microscopic nanocrystals of metallic silver are released from the dressing onto the wound bed. The silver has an antimicrobial action which destroys a range of bacteria, including both Grampositive and Gram-negative bacteria [22].

SEM images of this product showed that the polyethylene fibers are coated with Ag. The coating appears homogeneous and uniform. The size of the Ag nanocrystals was determined at a magnification of 50,000; the images show that the particle size ranges from 200 to $450 \mathrm{~nm}$.

Moreover, its cytotoxicity was tested in vitro and in vivo on human. In the present work the studies are carried on focusing on the intracellular behavior of Ag nanoparticles released form acticoat during a wound dressing.

\section{Material and Methods}

2.1. Human Skin Samples. Patients were eligible for the study if recruited $<24 \mathrm{~h}$ postburn injury and were affected by partial-thickness burns. Patients were excluded if they were affected by full thickness burns or had a compromised immune system or were known to be hypersensitive to silver and its compounds.

Patients were also excluded in case of comorbidity (e.g., diabetes and cardiac or renal disease), chemical or electrical burns, multiple trauma, or were aged $<5$ or $>60$. Skin biopsies were obtained from a set of eligible patients who gave consent for taking biopsy materials for scientific purposes, and the study was performed in compliance with the Declaration of Helsinki ethical guidelines.

Biopsies were collected by using punches of $4 \mathrm{~mm}$ inner diameter $7 \mathrm{~mm}$ depth. After seven days of treatment at dressing removal, two more duplicates were taken, one from the healed area and another from an unhealed zone. After 10 more days of treatment with a new dressing, another duplicate sample was taken from the newly healed area.

2.2. TEM. The samples were preserved in a $2.5 \%$ glutaraldehyde $/ 0.1 \mathrm{M}$ sodium cacodylate buffer overnight at $4^{\circ} \mathrm{C}$. The samples were then treated with $1 \%$ OsO4/0.1 M sodium cacodylate buffer and dehydrated using ethanol solutions of increasing concentrations before embedding in EPON epoxy resins. Ultrathin sections (ultramicrotome, LKB, Stockholm, Sweden) were obtained and treated with $1 \%$ uranyl acetate and $1 \%$ lead citrate. The samples were analyzed by TEM (Electronic Microscopy Service, Department of Biology, University of Padova, Padua, Italy) using a Tecnai G12 electron microscope (FEI, acceleration voltage $100 \mathrm{kV}$ ). The image acquisition system consisted of a video camera, TIETZ (Tietz Video and Image Processing Systems GmbH, Gauting, Germany), and the TIA FEI Imaging Software (FEI Company, Hillsboro, OR, USA).

2.3. Cell Cultures. Human dermal fibroblasts were prepared according to a modified version of the Rheinwald and Green protocol. After epithelial sheet dispase removal, the dermis was cut into small pieces $\left(2-3 \mathrm{~mm}^{2}\right)$, and fibroblasts were isolated by sequential digestion with $0.25 \% \mathrm{w} / \mathrm{v}$ trypsin for $20 \mathrm{~min}$ and $0.25 \% \mathrm{w} / \mathrm{v}$ collagenase for $4 \mathrm{~h}$. These cells were then cultured with Dulbecco's Modified Eagle Medium (DMEM), (Lonza S.r.l., Milano, Italy) supplemented with $10 \%$ fetal bovine serum (FBS) (Bidachem S.p.A., Milano, Italy) and 100 units $/ \mathrm{mL}$ penicillin and $100 \mu \mathrm{g} / \mathrm{mL}$ streptomycin to form complete DMEM (cDMEM). The medium was changed twice a week, and the cells were harvested by trypsin treatment. After detachment from culture plates, fibroblasts were cultured in 3D collagen-based scaffolds (MatriDerm, Dr. Suwelack Skin and Health Care AG, Billerbeck, Germany) at a density of $10^{5}$ cells $/ \mathrm{cm}^{2}$, obtaining a reconstructed dermallike tissue in vitro. Cells were grown in the 3D scaffold for 10 
days in $800 \mu \mathrm{L}$ of cDMEM. The Ag NP-based dressing was applied above the $3 \mathrm{D}$ cell cultures.

2.4. MTT Assay. To determine the kinetics of cell growth with or without Ag NPs, the MTT-based (methyl-thiazolyltetrazolium) cytotoxicity assay was performed according to the method of Denizot and Lang with minor modifications. This colorimetric assay is an indirect method for assessing cell growth and proliferation. MTT gives a yellowish aqueous solution, which, on reduction with dehydrogenases or reducing agents present in metabolically active cells, yields a violet-blue water insoluble dye compound, formazan. The lipid soluble formazan is extracted with organic solvents and quantified spectrophotometrically. The amount of MTT formazan produced is directly proportional to the metabolic activity of cells. After harvesting the culture medium, the cells were incubated for $3 \mathrm{~h}$ at $37^{\circ} \mathrm{C}$ in $1 \mathrm{~mL}$ of $0.5 \mathrm{mg} / \mathrm{mL}$ MTT solution prepared in phosphate buffer saline solution (PBS). After removal of the MTT solution by pipette, $0.5 \mathrm{~mL}$ of $10 \%$ dimethyl sulfoxide in isopropanol (iDMSO) was added to extract the formazan in the samples for $30 \mathrm{~min}$ at $37^{\circ} \mathrm{C}$. For each sample, absorbance values at $570 \mathrm{~nm}$ were recorded in duplicate on $200 \mu \mathrm{L}$ aliquots deposited in microwell plates using a multilabel plate reader (Victor 3 Perkin Elmer, Milano, Italy). The mitochondrial functionality in the Ag NPtreated cells is calculated as the ratio between the absorbance at $570 \mathrm{~nm}$ of the treated sample and the absorbance of a control sample expressed as a percentage.

2.5. Morphological Analysis. Concurrently with the MTT assay, morphological analyses were carried out on the duplicate samples. After removing the Ag NP-based dressing and the culture medium, the remaining dermal-like tissue was embedded in Optimal Cutting Temperature (OCT) compound, frozen in liquid nitrogen and preserved at $-80^{\circ} \mathrm{C}$ until cutting. Tissue sections ( $>7 \mu \mathrm{m}$ thickness) were obtained using a cryostat (CM1950, Leica, Milano, Italy) and deposited onto gelatin-coated glass slides. They were fixed with absolute acetone for $10 \mathrm{~min}$ at room temperature and cryopreserved at $-20^{\circ} \mathrm{C}$ until use. In order to visualize the cell distribution inside the scaffold and to investigate the possibility of nuclear fragmentation, the fibroblasts nuclei were stained with H33342 fluorochrome (Sigma Aldrich, Milano, Italy, final concentration of $2 \mu \mathrm{g} / \mathrm{mL}$ ). The samples were observed using a Zeiss Axioplan fluorescence microscope equipped with a digital camera (DC500, Leica, Milano, Italy).

In order to quantify the number of live cells and highlight the presence of apoptotic cells, a parallel set of in vitro experiments were carried out. Hoechst H33342 dye was added to the 3D dermal-like cell culture simultaneously with YOPRO-1 iodide dye (excitation wavelength $491 \mathrm{~nm} /$ emission wavelength $509 \mathrm{~nm}$, Molecular Probes). Hoechst H33342 dye stains the nuclei in the whole population of cells, while YO-PRO-1 stains specifically the apoptotic cells. YO-PRO1 is a green fluorescent probe, which can enter cells once their plasma membrane has reached a certain degree of permeability.

The cell membrane during apoptosis becomes slightly permeable and YO-PRO-1 can freely enter the cell and bind

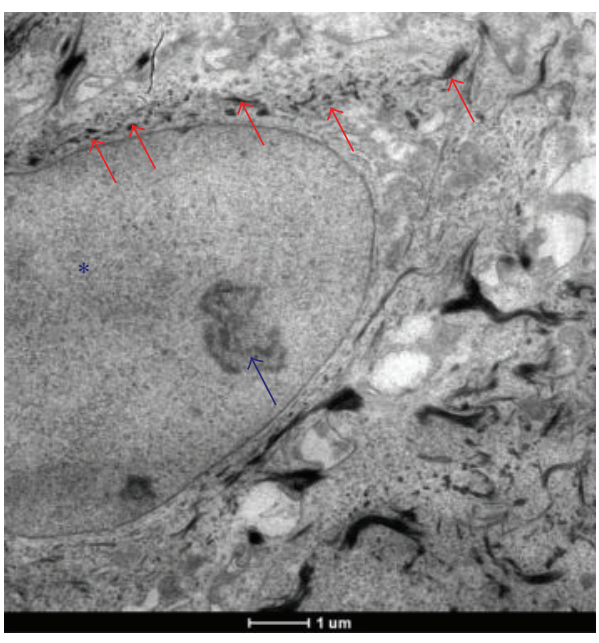

FIGURE 1: TEM images of a fibroblast present in a healed skin sample treated with AgNP based medical devices. The cells are healthy as the right morphological features show: integrity of plasma membrane, nuclear integrity (blue ${ }^{*}$ ), and active nucleolus (blue arrow).

to its nucleic acids, enhancing its fluorescence intensity. The number of different cells was counted, and live cells are calculated as the difference between the number of cells stained with Hoechst H33342 and the number of apoptotic cells stained with YO-PRO-1. Immediately after the removal of the Ag NP-based dressing from the 3D cell cultures, Hoechst 33342 and YO-PRO-1 were added to the cell cultures. The cells were incubated at $37^{\circ} \mathrm{C}$ for one hour, and then, the culture multiwell plate containing the cells was transferred to a confocal laser scanning microscope to monitor YO-PRO-1 and Hoechst fluorescence.

A fluorescence confocal laser scanning microscope (Axiovert 100M, Zeiss, Germany) with a $10^{\circ}$ magnification objective was used for the detection of Hoechst H33342 and YO-PRO-1 stained cells. The fluorescent dye, YO-PRO-1, was excited with a $25 \mathrm{~mW}$ Argon laser at $488 \mathrm{~nm}$. Emission was recorded above $510 \mathrm{~nm}$. The Hoechst $\mathrm{H} 33342$ fluorescence was detected at $460 \mathrm{~nm}$ after excitation at $346 \mathrm{~nm}$. The microscope was equipped with a motorized stage, and the LSM 510 (Zeiss) software enabled memorization of stage positions. For each sample, images were taken at the preset stage positions at various depths.

2.6. ROS Measurements. The OxiSelect ROS Assay Kit is a cell-based assay for measuring hydroxyl, peroxyl, and other reactive oxygen species activity within a cell. The assay employs the cell-permeable fluorogenic probe DCFH$\mathrm{DA}$, which diffuses into cells and is deacetylated by cellular esterases into the nonfluorescent DCFH (Figure 1). In the presence of ROS, DCFH is rapidly oxidized to highly fluorescent DCF. Fluorescence is read on a standard fluorometric plate reader.

\section{Results and Discussion}

fibroblast present in the deeper layer of burned skin are able to uptake AgNps (Figure 1, red arrows). The cells are 


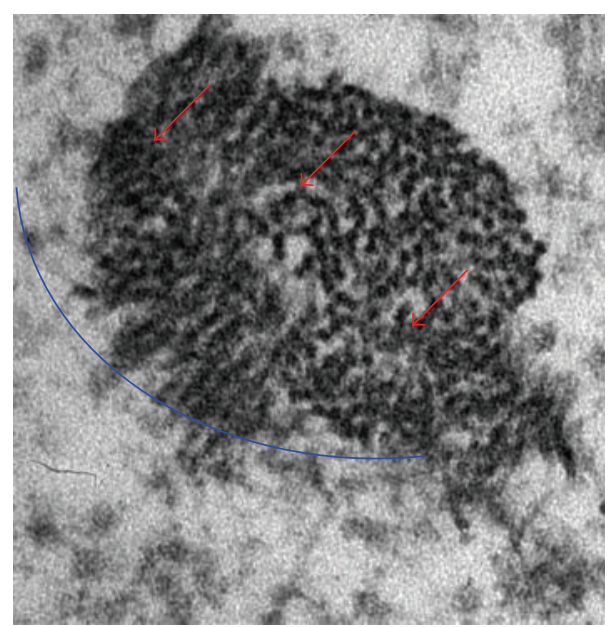

FIGURE 2: TEM images of a fibroblast present in a healed skin sample treated with AgNP based medical devices. Nanoparticles taking up by endocytosis. AgNps in normal human dermal fibroblasts take up AgNps (red arrows) by endocytosis (blue arc).

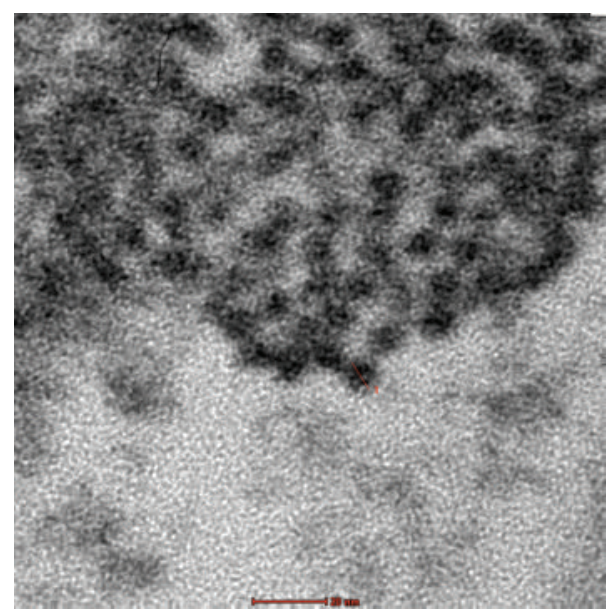

FIgURE 3: TEM images of AgNps (red arrows) in cytoplasmatic space of fibroblast present in a healed skin sample treated with AgNP based medical devices. The diameter of particles released from the medical devices are of $20 \mathrm{~nm}$, indicating that their aggregates are able to enter the cells by endocytosis.

healthy as the right morphological features show: integrity of plasma membrane, nuclear integrity (blue*), and active nucleolus (blue arrow). All nanoparticles are taken up by mammalian cells by such mechanisms as pinocytosis, endocytosis dependent on caveolae and lipid raft composition, clathrin-dependent endocytosis and phagocytosis [10], endocytosis $\{$ Endo (within) cytosis (cell) $\}$, is a process in which a substance gains entry into a cell without passing through the cell membrane. This process is subdivided into three different types: pinocytosis, phagocytosis, receptor mediatedendocytosis. Receptor mediated endocytosis is an endocytotic mechanism in which specific molecules are ingested into the cell. The specificity results from a receptor-ligand interaction. Receptors on the plasma membrane of the target tissue will

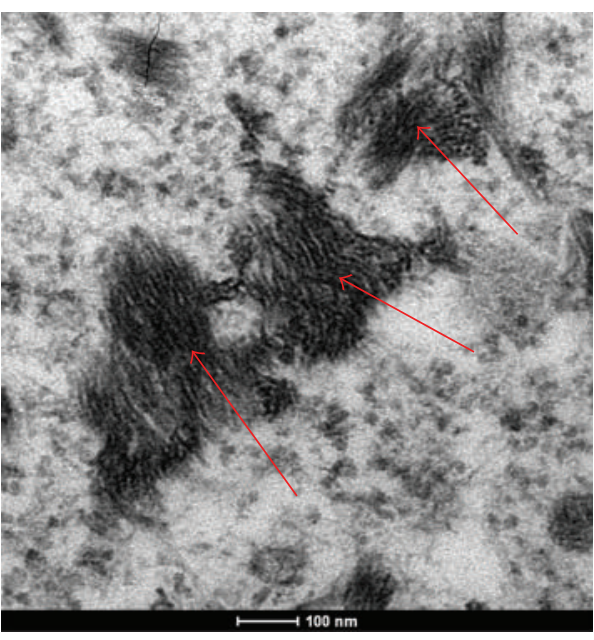

FIgURE 4: TEM images of AgNps (red arrows) in cytoplasmatic space of fibroblast present in a healed skin sample treated with AgNP based medical devices.

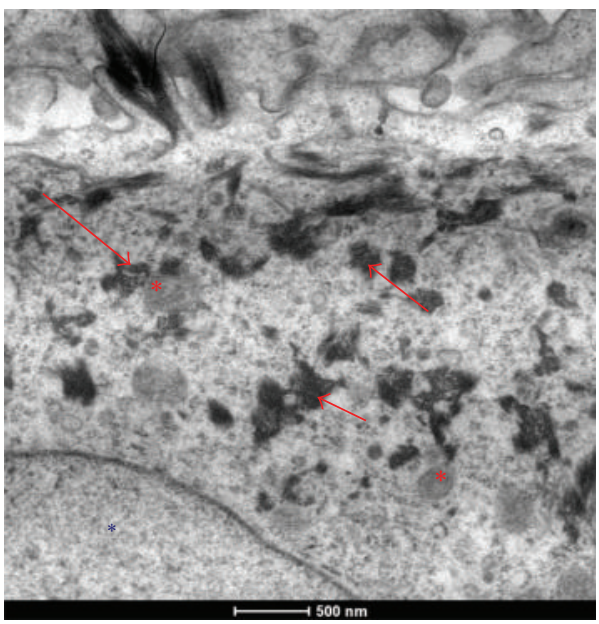

FIGURE 5: TEM images of AgNps (red arrows) in cytoplasmatic space of fibroblast present in a healed skin sample treated with AgNP based medical devices. AgNP are closed to the mitochondria (red*); nuclei is indicated by blue* .

specifically bind to ligands on the outside of the cell. An endocytotic process occurs, and the ligand is ingested. In each case, endocytosis results in the formation of an intracellular vesicle by virtue of the invagination of the plasma membrane and membrane fusion.

AgNps are no exception in this respect; as shown in Figure 2, indeed, normal human dermal fibroblasts take up AgNps (red arrows) by endocytosis (blue arc). As well reported in Figure 3 the diameter of particles released from the medical devices is of $20 \mathrm{~nm}$, (note: a fibroblast size is about $100 \mu \mathrm{m}$ ) indicating that their aggregates are able to enter into the cells by endocytosis.

As regards intracellular localization of AgNps, their ability to form aggregates of about $200 \mathrm{~nm}$ (Figure 4, red arrows) was confirmed. They are absent from the cell nucleus, endoplasmic reticulum, or Golgi complex (Figure 5). They 


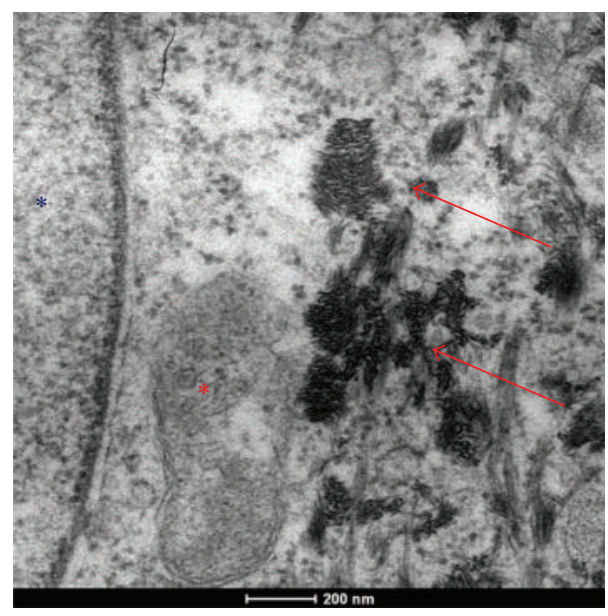

(a)

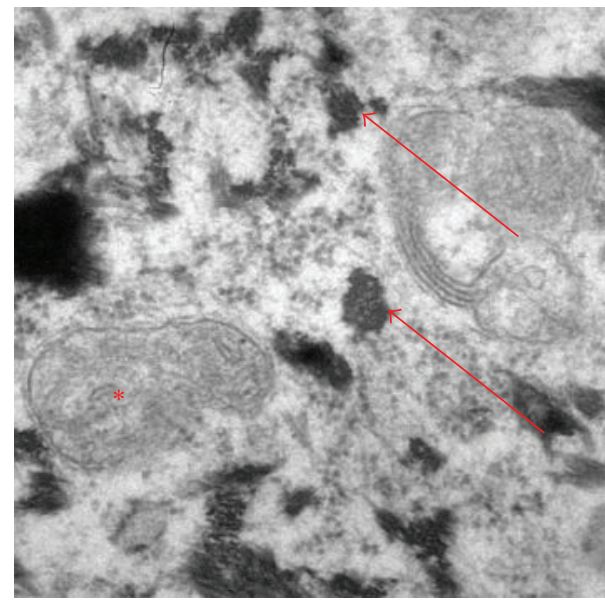

(b)

FIgURE 6: TEM images of AgNps (red arrows) in cytoplasmatic space of fibroblast present in a healed skin sample treated with AgNps based Medical devices. AgNP are closed to the mitochondria $\left(\operatorname{red}^{*}\right)$; and not inside the mitochondria and inside the nuclei (indicated by blue ${ }^{*}$ ).

also formed agglomerates in the perinuclear region (Figure 5, red arrows). The transmission electron microscopy (TEM) analysis indicated the absence of AgNps (Figures 5 and 6 red arrows) inside the mitochondria (Figures 5 and 6 red $^{*}$ ) and nucleus (Figure 5 blue $^{*}$ ). AgNps are closed to the outer membrane of the mitochondria as it is well shown in Figure 7 red arrows (mitochondria red*). Mitochondria are organized in the perinuclear zone of the cytoplasm (Figure 8 , square barked red), recruiting the larger quantity of AgNps (Figure 8 red arrows) present in the cytoplasm.

In these conditions, mitochondria are small (Figure 8 red $^{*}$ ), round and in high number.

To investigate if Ag NPs could negatively affect cell survival, we evaluated their toxicity on fibroblasts in vitro. A collagen-based scaffold was employed as a support for a 3D cell culture of fibroblasts to obtain a dermal-like tissue. Morphological analyses were carried out to investigate nuclei morphology and cellular distribution within the scaffold. As

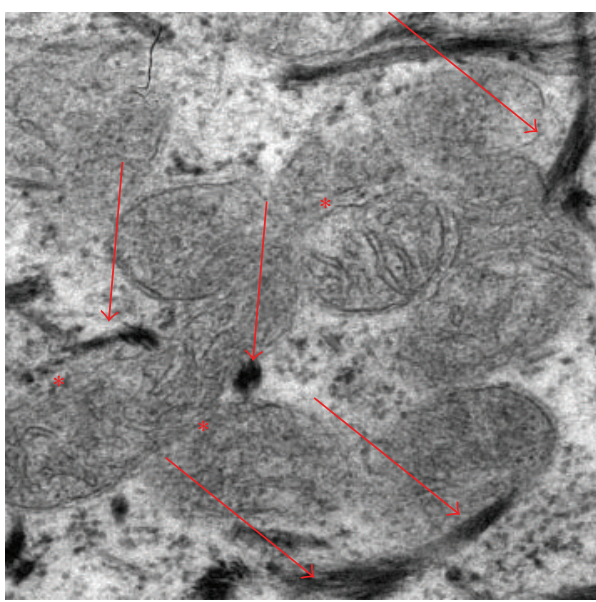

FIgURE 7: TEM images of AgNps (red arrows) in cyplosmasmatic space of fibroblast present in a healed skin sample treated with AgNP based Medical devices. AgNP (red arrows) are closed to the mitochondria $\left(\right.$ red $\left.^{*}\right)$.

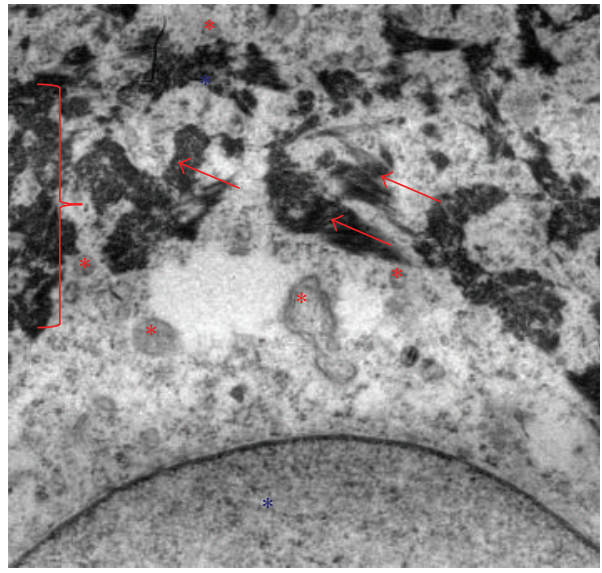

FIgURE 8: TEM images of AgNps (red arrows) in cytoplasmatic space of fibroblast present in a healed skin sample treated with AgNP based Medical devices. AgNps are closed to the outer membrane of the mitochondria that are organized in the perinuclear zone of the cytoplasm (square barked red), recruiting the larger quantity of AgNps present in the cytoplasm.

reported in Figure 9(a) the dermal-like tissue appears as a multilayer of cells, where the fibroblasts are able to proliferate and fill the scaffolds during the course of the experiments. No signs of apoptosis were detected. The similar distribution of cells was seen in the Ag NP-treated samples (Figure 9(b)). Interestingly, despite the reduced mitochondrial functionality observed, the nuclei are still present and appear to be undamaged. There was no observable presence of apoptotic bodies or nuclear fragmentation.

A quantitative comparison of the number of live cells in the treated and untreated 3D cell cultures is reported in Figure 10(a). The results show that the number of live cells increased with time at the same rate in both samples. The YOPRO-1 assay showed that there were no apoptotic cells visible in the sample treated with Ag NPs. 


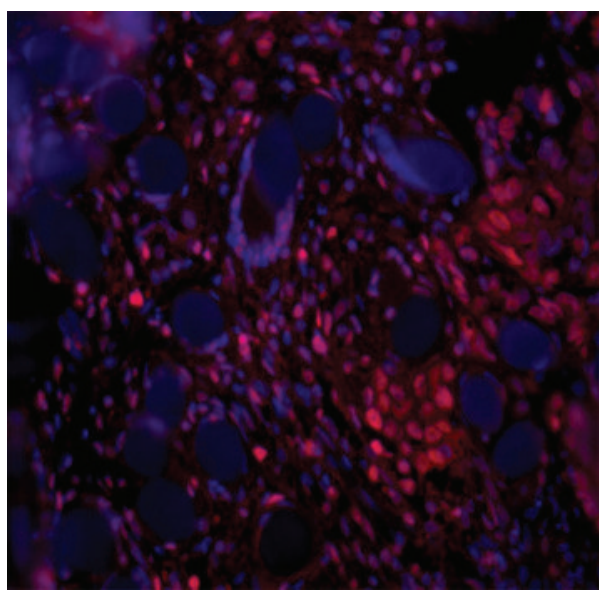

(a)

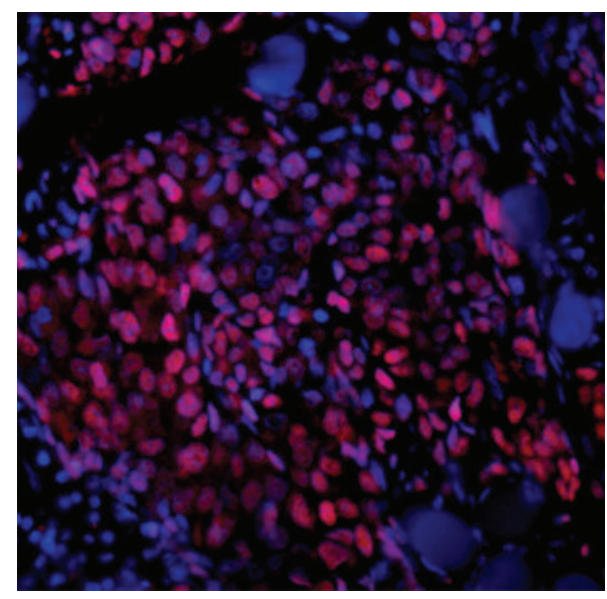

(b)

FIGURE 9: Dermal-like tissue reconstructed in vitro. Cells, visible thanks to the red staining of the nuclei, can be seen inside the collagen-based scaffold and appear to be organized in layers. (a) Un-treated control after 9 days from the beginning of the experiments; (b) treated control at nine days.

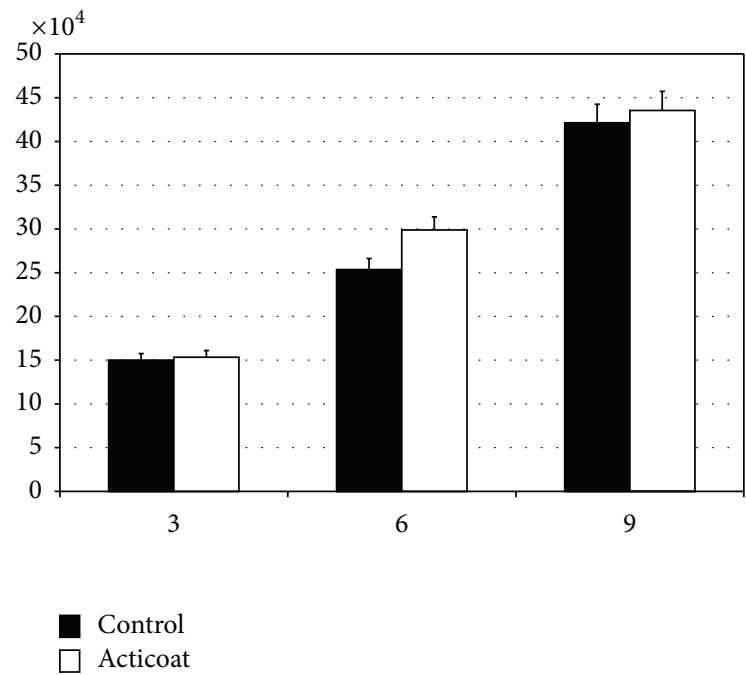

(a)

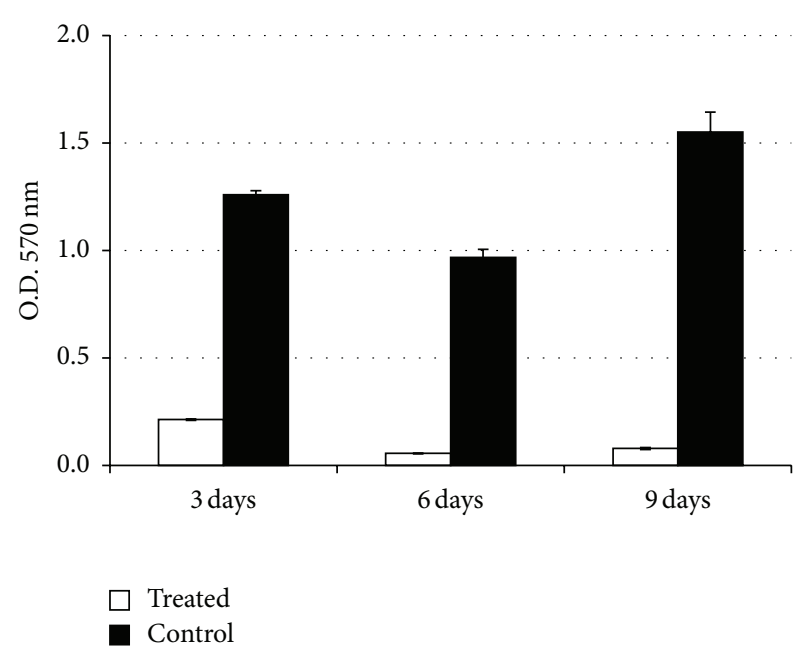

(b)

FIGURE 10: (a) Progression of cell growth in time in a 3D dermal-like tissue after Ag NP streatment (white) and in the control sample (black), mean value \pm SD samples versus time. The count of the live cells in the sample is obtained as the sum of the live cells at various depths at each position. (b) MTT test for mitochondrial activity in a 3D dermal-like tissue after Ag NPs treatment (white) and in the control sample (black) mean value \pm SD samples versus time. As it is well evident, when silver nanoparticles are present, no mitochondrial activity is detectable.

At three, six, and nine days, MTT assays were carried out to assess the mitochondrial function in cells treated with Ag NPs. Cytotoxicity in vitro is usually estimated with the use of colorimetric tests; their principle is the reduction of tetrazolium salts, MTT (3-(4,5-dimethylthiazol-2-yl)-2,5diphenyltetrazolium bromide) to formazan. The reduction is carried out by a mitochondrial reductase and is an indirect measure of cell population viability.

As reported in Figure 10(b), a time-dependent decrease in metabolic activity was observed in the cells treated with the Ag NP-based dressing. This confirms the ability of AgNPs to impair mitochondrial function. Examples of MTT test application for AgNps cytotoxicity (ROS) are present in every cell, being produced by the mitochondrial and cytoplasmic oxidation processes. Under environmental stress, the cell reacts by increased ROS generation, and this leads to imbalance between ROS generation and their neutralization by antioxidative enzymes and low molecular weight antioxidants, among others by glutathione. This disturbance of the redox equilibrium is defined as oxidative stress. Under conditions of oxidative stress the cell accumulates ROS, and the antioxidative response that follows involves modifications in signaling pathways.

In order to test if this damage could be correlated to a ROS production we tested ROS generations. As reported in Figure 11 there is a ROS production in presence of AgNPs. 


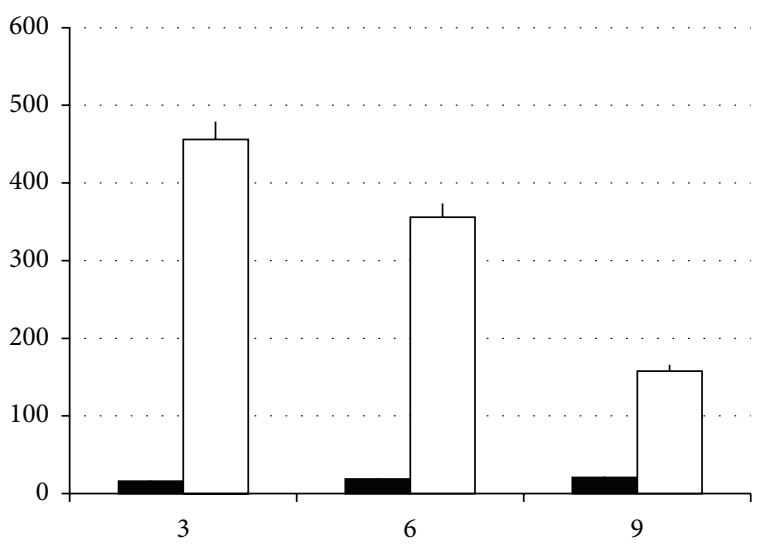

Control

Acticoat

FIGURE 11: The ROS measurement in dermal-like tissue reconstructed in vitro with (white) and without NgNPs (black) treatment. As it is well evident, when silver nanoparticles are present, ROS activity is detectable.

This production decreases in time, according to the ability of the mitochondria to capture AgNPs.

\section{Conclusions}

ROS increase due to nanoparticle treatment has been shown to be the key factor in the biological effects in vivo and in vitro [23-25]. From our TEM microphotographs it can be judged that AgNps accumulate outside the mitochondria. It is possible that this is the direct cause of mitochondrial damage and the disturbed function of the respiratory chain resulting in ROS generation and oxidative stress. No AgNps were detected inside the nucleus, and no fragmented nuclei were observed. Despite the presence of AgNps inside the cytoplasm, the nuclear membrane is intact and is round in shape. The nucleolus visible confirms that the chromatin is not condensed, but that it has a structure that allows transcription to proceed. We hypothesize that once the AgNps have been released into the cytoplasm, they generate ROS.

We speculate, then, that mitochondria are moved around the nucleus to act as a physical-chemical barrier to prevent ROS and AgNps from reaching the nuclear membrane. If the mitochondrial membrane breaks down due to the action of ROS, antioxidative enzymes, such as mitochondrial superoxide dismutase (mtSOD), catalase, glutathione peroxidase, and thioredoxin peroxidase $[26,27]$, are released from the mitochondria into the cytoplasm to quench the ROS.

\section{Conflict of Interests}

The authors declare that there is no conflict of interests.

\section{References}

[1] S. Sivolella, E. Stellini, G. Brunello et al., "Silver nanoparticles in alveolar bone surgery devices and toxicity of silver nanoparticles in alveolar bone surgery devices," Journal of Nanomaterials, vol. 2012, Article ID 975842, 12 pages, 2012.

[2] R. Cortivo, V. Vindigni, L. Iacobellis, G. Abatangelo, P. Pinton, and B. Zavan, "Nanoscale particle therapies for wounds and ulcers," Nanomedicine, vol. 5, no. 4, pp. 641-656, 2010.

[3] N. Silvestry-Rodriguez, E. E. Sicairos-Ruelas, C. P. Gerba, and K. R. Bright, "Silver as a disinfectant," Reviews of Environmental Contamination and Toxicology, vol. 191, pp. 23-45, 2007.

[4] A. Melaiye and W. J. Youngs, "Silver and its application as an antimicrobial agent," Expert Opinion on Therapeutic Patents, vol. 15, no. 2, pp. 125-130, 2005.

[5] B. S. Atiyeh, M. Costagliola, S. N. Hayek, and S. A. Dibo, "Effect of silver on burn wound infection control and healing: review of the literature," Burns, vol. 33, no. 2, pp. 139-148, 2007.

[6] J. Jain, S. Arora, J. M. Rajwade, P. Omray, S. Khandelwal, and K. M. Paknikar, "Silver nanoparticles in therapeutics: development of an antimicrobial gel formulation for topical use," Molecular Pharmaceutics, vol. 6, no. 5, pp. 1388-1401, 2009.

[7] L. B. Rice, "The clinical consequences of antimicrobial resistance," Current Opinion in Microbiology, vol. 12, no. 5, pp. 476481, 2009.

[8] I. Tocco, B. Zavan, F. Bassetto, and V. Vindigni, "Nanotechnology-based therapies for skin wound regeneration," Journal of Nanomaterials, vol. 2012, Article ID 714134, 11 pages, 2012.

[9] M. Quirynen, M. de Soete, and D. van Steenberghe, "Infectious risks for oral implants: a review of the literature," Clinical Oral Implants Research, vol. 13, no. 1, pp. 1-19, 2002.

[10] M. Esposito, H. V. Worthington, V. Loli, P. Coulthard, and M. G. Grusovin, "Interventions for replacing missing teeth: antibiotics at dental implant placement to prevent complications," Cochrane Database of Systematic Reviews, vol. 7, Article ID CD004152, 2010.

[11] D. Kinane and P. Bouchard, "Periodontal diseases and health: consensus Report of the Sixth European Workshop on Periodontology," Journal of Clinical Periodontology, vol. 35, no. 8, pp. 333-337, 2008.

[12] G. Chiara, F. Letizia, F. Lorenzo et al., "Nanostructured biomaterials for tissue engineered bone tissue reconstruction," International Journal of Molecular Sciences, vol. 13, no. 1, pp. 737757, 2012.

[13] R. P. Allaker, "Critical review in oral biology \& medicine: the use of nanoparticles to control oral biofilm formation," Journal of Dental Research, vol. 89, no. 11, pp. 1175-1186, 2010.

[14] D. R. Monteiro, L. F. Gorup, A. S. Takamiya, A. C. Ruvollo-Filho, E. R. D. Camargo, and D. B. Barbosa, "The growing importance of materials that prevent microbial adhesion: antimicrobial effect of medical devices containing silver," International Journal of Antimicrobial Agents, vol. 34, no. 2, pp. 103-110, 2009.

[15] X. Wu, J. Li, L. Wang, D. Huang, Y. Zuo, and Y. Li, “The release properties of silver ions from Ag-nHA/TiO $/ 2$ PA66 antimicrobial composite scaffolds," Biomedical Materials, vol. 5, no. 4, Article ID 044105, 2010.

[16] J. Li, Y. Zuo, Y. Man et al., "Fabrication and biocompatibility of an antimicrobial composite membrane with an asymmetric porous structure," Journal of Biomaterials Science, Polymer Edition, vol. 23, no. 1-4, pp. 81-96, 2012.

[17] O. D. Schneider, D. Mohn, and R. Fuhrer, "Biocompatibility and bone formation of flexible, cotton wool-like PLGA/calcium 
phosphate nanocomposites in sheep," The Open Orthopaedics Journal, vol. 5, pp. 63-71, 2011.

[18] J. Ye, Q. Yao, A. Mo et al., "Effects of an antibacterial membrane on osteoblast-like cells in vitro," International Journal of Nanomedicine, vol. 6, pp. 1853-1861, 2011.

[19] M. Chiapasco and M. Zaniboni, "Clinical outcomes of GBR procedures to correct peri-implant dehiscences and fenestrations: a systematic review," Clinical Oral Implants Research, vol. 20, no. 4, pp. 113-123, 2009.

[20] C. Rigo, L. Ferroni, I. Tocco et al., "Active silver nanoparticles for wound healing," International Journal of Molecular Sciences, vol. 14, no. 3, pp. 4817-4840, 2013.

[21] C. Rigo, M. Roman, I. Munivrana et al., "Characterization and evaluation of silver release from four different dressings used in burns care," Burns, vol. 38, no. 8, pp. 1131-1142, 2012.

[22] H. Q. Yin, R. Langford, and R. E. Burrell, "Comparative evaluation of the antimicrobial activity of ACTICOAT* Antimicrobial Barrier Dressing," Journal of Burn Care and Rehabilitation, vol. 20, no. 3, pp. 195-200, 1999.

[23] S. Kim, J. E. Choi, J. Choi et al., "Oxidative stress-dependent toxicity of silver nanoparticles in human hepatoma cells," Toxicology In Vitro, vol. 23, no. 6, pp. 1076-1084, 2009.

[24] C. Greulich, J. Diendorf, T. Simon, G. Eggeler, M. Epple, and M. Köller, "Uptake and intracellular distribution of silver nanoparticles in human mesenchymal stem cells," Acta Biomaterialia, vol. 7, no. 1, pp. 347-354, 2011.

[25] P. Aguiari, S. Leo, B. Zavan et al., "High glucose induces adipogenic differentiation of muscle-derived stem cells," Proceedings of the National Academy of Sciences of the United States of America, vol. 105, no. 4, pp. 1226-1231, 2008.

[26] H. C. Lee, P. H. Yin, C. Y. Lu, C. W. Chi, and Y. H. Wei, "Increase of mitochondria and mitochondrial DNA in response to oxidative stress in human cells," Biochemical Journal, vol. 348, no. 2, pp. 425-432, 2000.

[27] S. Marchi, C. Giorgi, J. M. Suski et al., "Mitochondria-ros crosstalk in the control of cell death and aging," Journal of Signal Transduction, vol. 2012, Article ID 329635, 17 pages, 2012. 


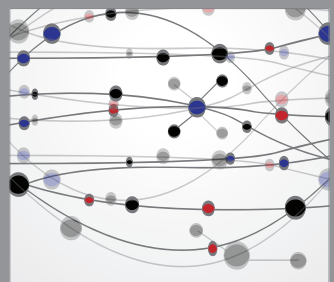

The Scientific World Journal
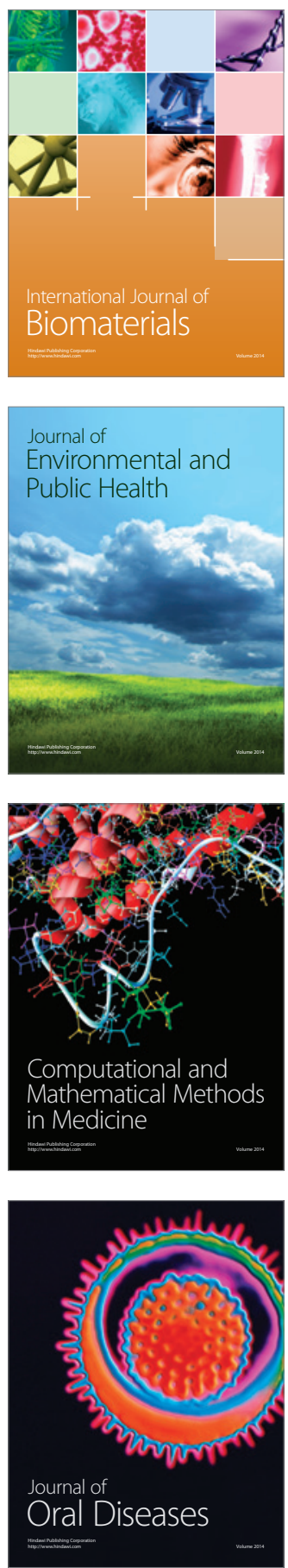
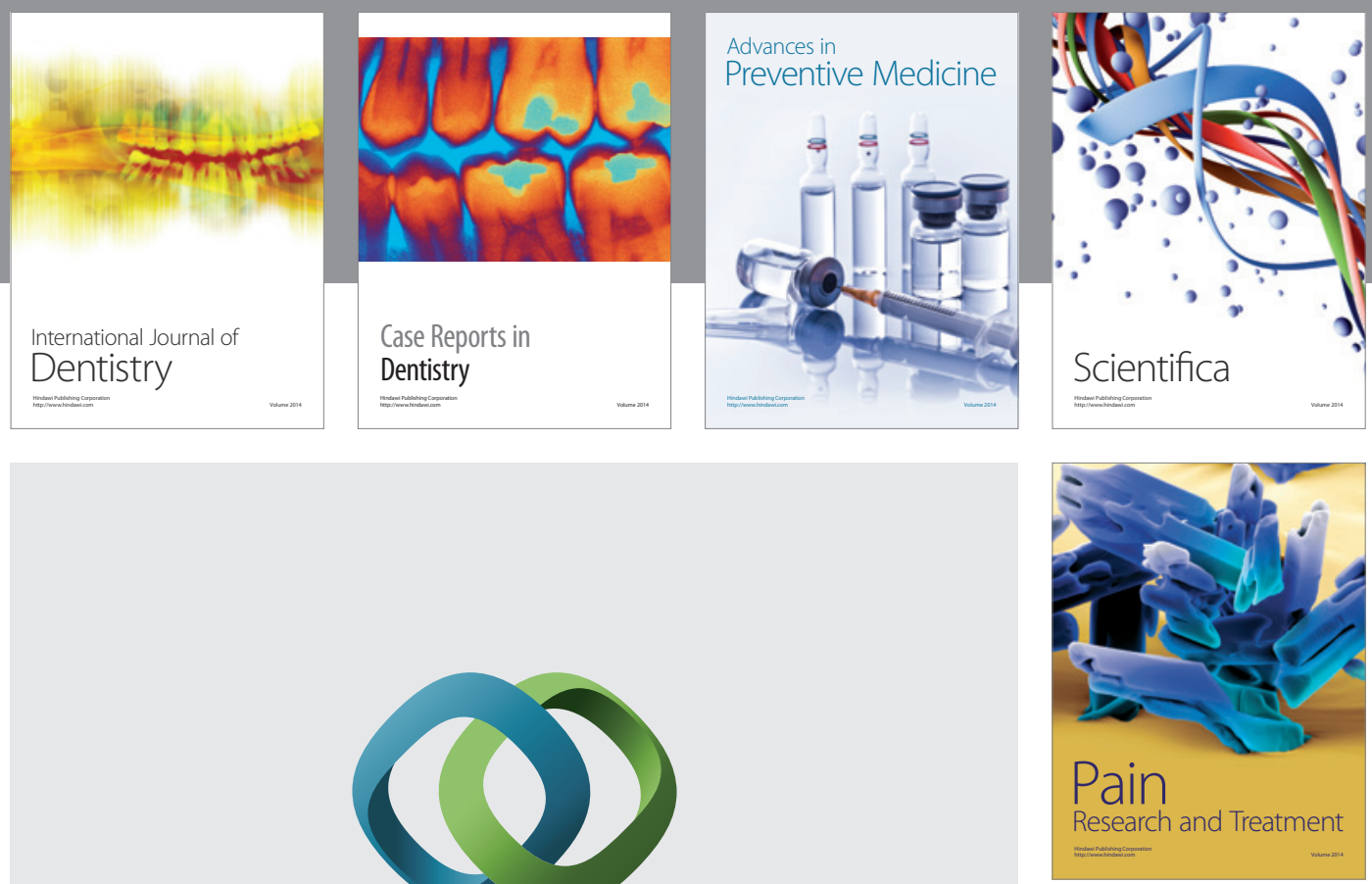

\section{Hindawi}

Submit your manuscripts at

http://www.hindawi.com
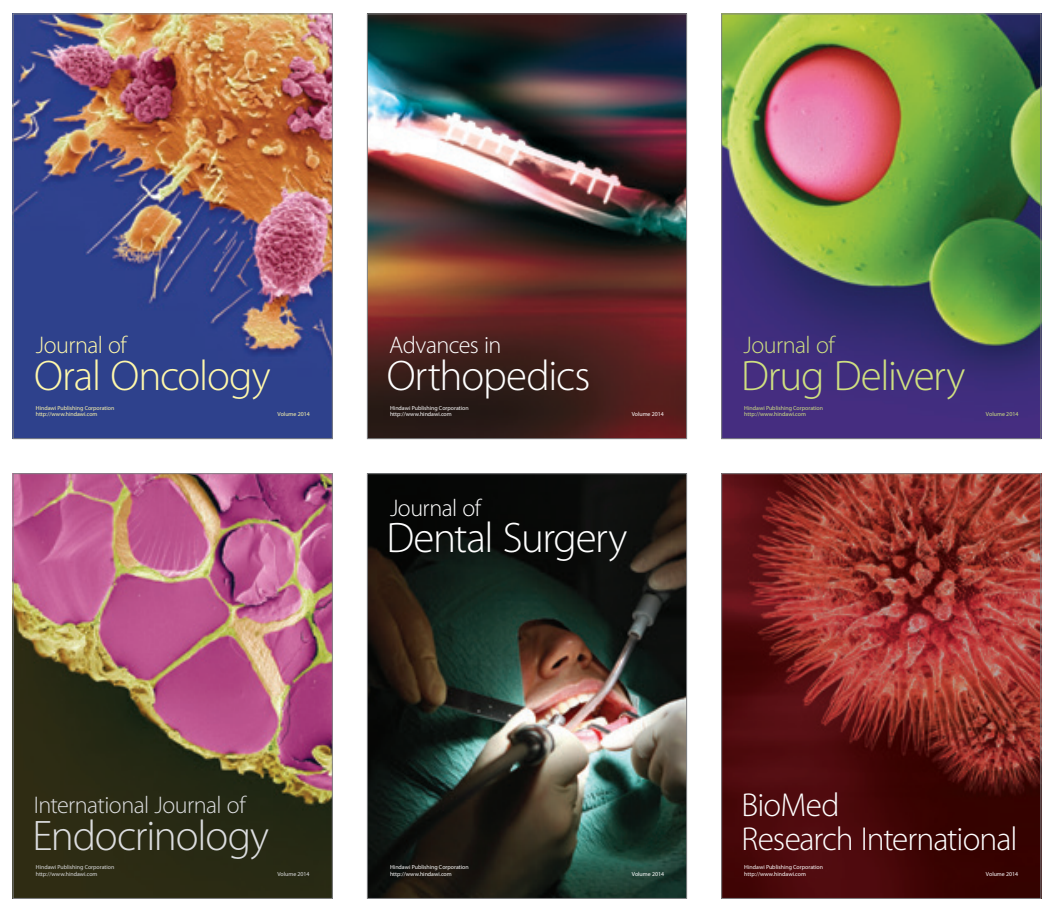

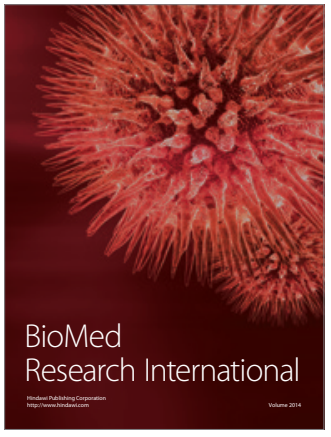

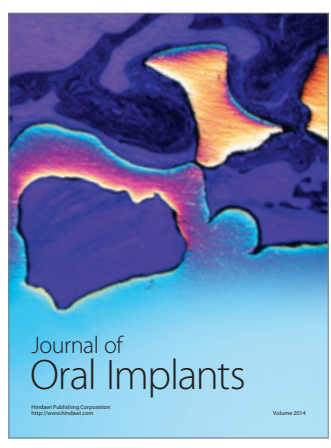
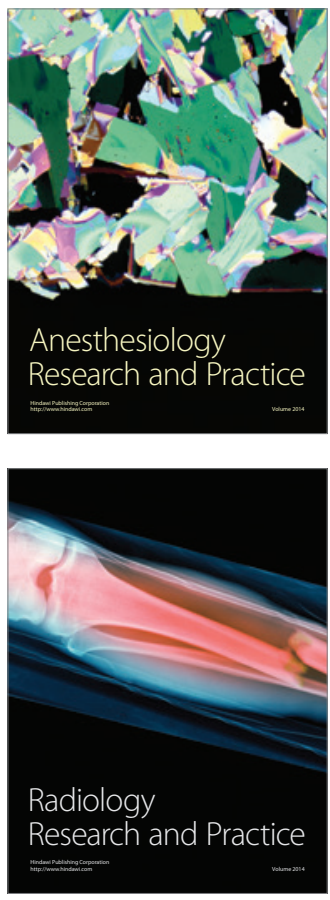